\title{
Does relocating information in text depend on verbal or visuospatial abilities? An individual-differences analysis
}

\author{
KATHERINE A. RAWSON and AKIRA MIYAKE \\ University of Colorado, Boulder, Colorado
}

\begin{abstract}
In this individual-differences study, we evaluated the prevalent view that relocating information in a previously read text depends primarily on visuospatial abilities. Participants read a text, answered fillin-the-blank test questions, and identified which page and line in the original text contained the sentence in each question. They also completed a battery of verbal and visuospatial tasks. Performance on verbal tasks was highly predictive of the accuracy of both page and line identifications, and this correlation remained significant even after we controlled for performance on visuospatial tasks. In contrast, performance on visuospatial tasks was not predictive of either page or line identification accuracy, once verbal abilities were controlled for. These results suggest an important role for verbal abilities in relocation of text information and a lesser role for visuospatial abilities than has previously been assumed.
\end{abstract}

Most of us can remember a circumstance in which we have been asked a question (e.g., on an exam) to which we knew we had read the answer. On some occasions, we have even had the experience of knowing where to look in the text to find the information (e.g., "Oh, it was in the top corner, on the left page"). Consistent with this experience, previous research has shown that people can identify the location of information in a previously read text with above chance accuracy (Lovelace \& Southall, 1983; Zechmeister \& McKillip, 1972; Zechmeister, McKillip, Pasko, \& Bespalec, 1975). For example, Rothkopf (1971) had participants read a 12-page text and then answer short-answer questions. After each question, the participants were asked to indicate where in the original text the question information had appeared. Although not forewarned of this relocation task, they could identify, with above chance accuracy, both on which page and where on that page the target information had appeared.

The main question addressed in the present study is what cognitive abilities support relocation performance. Given that people often report being able to "see" where information was in a text, one possibility is that relocation of text information is supported primarily by visuospatial abilities. In fact, previous research has suggested that visuospatial location information for words is en-

\footnotetext{
This research was supported by an NSF Graduate Research Fellowship to the first author. We thank Mariarosa Gasbarro and Matt Jordan for assistance in data collection and scoring and Mike Emerson, John Dunlosky, and Jim Van Overschelde for helpful discussions and comments on earlier versions of this manuscript. Correspondence concerning this article should be addressed to K. Rawson, Department of Psychology, University of Colorado, 345 UCB, Boulder, CO 80309-0345 (e-mail: krawson@psych.colorado.edu).
}

coded and is available to readers for some time. In a study of garden path sentences, for example, Frazier and Rayner (1982) observed that readers sometimes know where their comprehension might have gone astray and can move their eyes directly back to the main source of the comprehension difficulty (see also Kennedy \& Murray, 1987). Consistent with such observations, most previous research on text relocation performance has explicitly assumed that readers rely on their memory for specific spatial locations of words and sentences in relocating text (Baccino \& Pynte, 1994, 1998; Lovelace \& Southall, 1983; Zechmeister \& McKillip, 1972; Zechmeister et al., 1975). If relocating information in text indeed depends on visuospatial information, readers with higher visuospatial abilities should be better at encoding and retrieving the spatial layout of the text and, hence, at relocating text as well.

Although this contribution of visuospatial abilities seems intuitive, an alternative possibility is that verbal abilities play an important role in relocating text information. If one has high verbal abilities, one is more likely to achieve a good understanding of the interrelationships among different ideas expressed in the text and the order in which they have been presented. Such information could then be used to infer where target information has appeared, without much reliance on visuospatial abilities per se. Although no direct evidence documents the role of verbal abilities in relocation performance at the text level, some recent research conducted at the sentence level suggests that much of the encoded information about the spatial locations of words may be short-lived and, consequently, that readers may rely on nonspatial information, such as temporal and item information, to reconstruct word locations within a sentence (Fischer, 
1999, 2000). Given that the processing of text takes place over a more extended time frame, it seems reasonable to hypothesize that readers who can comprehend the text and later reconstruct relevant text information well (i.e., those with higher verbal abilities) are also better at relocating text information.

In the present study, we evaluated the degree to which visuospatial abilities and verbal abilities contribute to text relocation performance from an individual-differences perspective, using a new virtual book methodology that simulated actual reading situations. Specifically, participants read a textbook passage from a virtual book, answered content questions, and then relocated the question information in the book. The participants also completed a battery of prevalently used verbal and visuospatial tasks tapping a wide range of abilities within each domain. Because verbal and visuospatial abilities are separable (Shah \& Miyake, 1996) but moderately correlated constructs (Carroll, 1993), we performed regression analyses to assess the unique contribution that each domain ability made to the prediction of relocation performance, controlling for the effect of a domain-general factor common to both. If relocation performance is supported primarily by visuospatial abilities, then visuospatial measures should predict individual differences in relocation performance. If it is supported primarily by verbal abilities, verbal measures should predict relocation performance.

\section{METHOD}

\section{Participants}

Fifty-eight undergraduates from the University of Colorado participated for course credit. All were native speakers of English.

\section{Relocation Task}

The experimental text was adapted from a chapter in a nutrition textbook discussing human aging. It was about 3,000 words in length, with the Flesch-Kincaid grade level of 10.8. The text contained three major sections of equivalent length, each preceded by a topic header (e.g., "What Is Aging and Why Does It Happen?"). Twenty-seven test items were developed using 9 sentences from each section. For each test item, 1-3 words were removed from the original sentence, and participants were required to fill in the missing words. The Appendix presents a text excerpt and corresponding test items.

At the beginning, the participants were told that they would read a text and then answer fill-in-the-blank questions. They were shown a short practice text in a manner similar to that for the experimental text (described below) and sample fill-in-the-blank questions to familiarize them with the task. Participants were not forewarned that they would also be asked to relocate text information. As is illustrated in the top of Figure 1, the experimental text was presented on 12 "pages" displayed on the computer screen, each containing 30 lines. Topic headers for the three subsections were presented in capital letters and were preceded and followed by a blank line. The pages were superimposed but with the outer margins exposed to simulate the visual cues that would be available when one is reading a textbook. Text presentation began with the virtual book "opened" to the first 2 pages, with all of the subsequent pages superimposed on the right side of the screen. After they had read the first 2 pages, the participants "turned" the page to reveal the next
2 pages by using the mouse to click on a screen button centered below the text. They were permitted to use either hand to control the mouse, but were not permitted to turn back in the text at any point during study. Because the screen button remained in the same spot during study, once the cursor had been placed on the button, a minimal amount of hand movement was required for one to advance the text. Thus, the encoding and maintenance of visuospatial information during study was unlikely to have been compromised by interference from manual movements.

After studying all 12 pages of the text, the participants were then informed about the relocation component of the test. Before completing the criterion test items, the participants practiced the relocation task with the practice text, and the experimenter verified that they completely understood the task. This practice took approximately $3 \mathrm{~min}$ to complete, including instructions. The participants were then presented with 27 test items, 1 at a time. For each item, they first typed in the missing word(s). They were then shown the virtual book and asked to relocate the corresponding sentence in the text. During this phase, all of the letters and symbols in the text (excluding punctuations) were replaced with the letter $\mathrm{X}$ in the appropriate case, as is illustrated in the bottom panel of Figure 1. The participants clicked on two buttons below the text to turn the pages forward and backward. After the participants had turned to the page they believed contained the sentence, they clicked on a line they believed included the sentence.

\section{Individual Difference Measures}

The verbal and visuospatial ability measures were selected to tap a wide range of abilities within each domain. Specifically, for each domain, we included a measure of working memory (reading span and Corsi blocks), a measure of long-term memory (narrative recall and Rey-Osterrieth Complex Figure Test), and a measure of reasoning and inference making (Nelson-Denny Reading Test and paper folding). For the verbal ability measures, we also included a common measure of verbal knowledge, a vocabulary test. The format and procedure for these tasks were as follows.

Verbal ability measures. In the reading span task (Daneman \& Carpenter, 1980), the participants were required to read aloud a set of 2-5 unrelated sentences presented one at a time and then recall the last word of each sentence. There were a total of 12 sentence sets, with 3 sets at each set size. In the narrative recall task, the participants were told to study a short, simple narrative (132 words in length, 5.3 Flesch-Kincaid grade level) for subsequent recall. After an 8-min filled interval, a free recall test for the content of the narrative was administered. The Nelson-Denny Reading Test (Brown, Bennett, \& Hanna, 1981) is a reading comprehension test that consists of 8 short expository texts, each accompanied by multiplechoice questions ( 36 total). Some of these questions were about specific facts mentioned in the text, and others required inference making. Fifteen minutes were allowed for answering as many questions as possible. The vocabulary test (Ekstrom, French, Harman, \& Derman, 1976) required the participants to select the closest synonym for a target word. They were given $8 \mathrm{~min}$ to answer 36 multiplechoice questions.

Visu ospatial ability measures. In the Corsi blocks task (the version developed by Miyake, Friedman, Rettinger, Shah, \& Hegarty, 2001), the participants were shown a set of 10 randomly arranged boxes and asked to remember the order in which they were "tapped" (shown as changing color, at the rate of $650 \mathrm{msec}$ per box). At the end of a sequence, they were to repeat the sequence by clicking on the squares in the same order. There were five trials at each sequence length (from three to eight). In the Rey-Osterrieth Complex Figure Test (Spreen \& Strauss, 1998), the participants were shown a complex line drawing and were asked to copy the figure onto a blank piece of paper. The figure was then removed, without any mention of a subsequent recall test. After a 10-min filled interval, the participants were given another blank piece of paper and were 

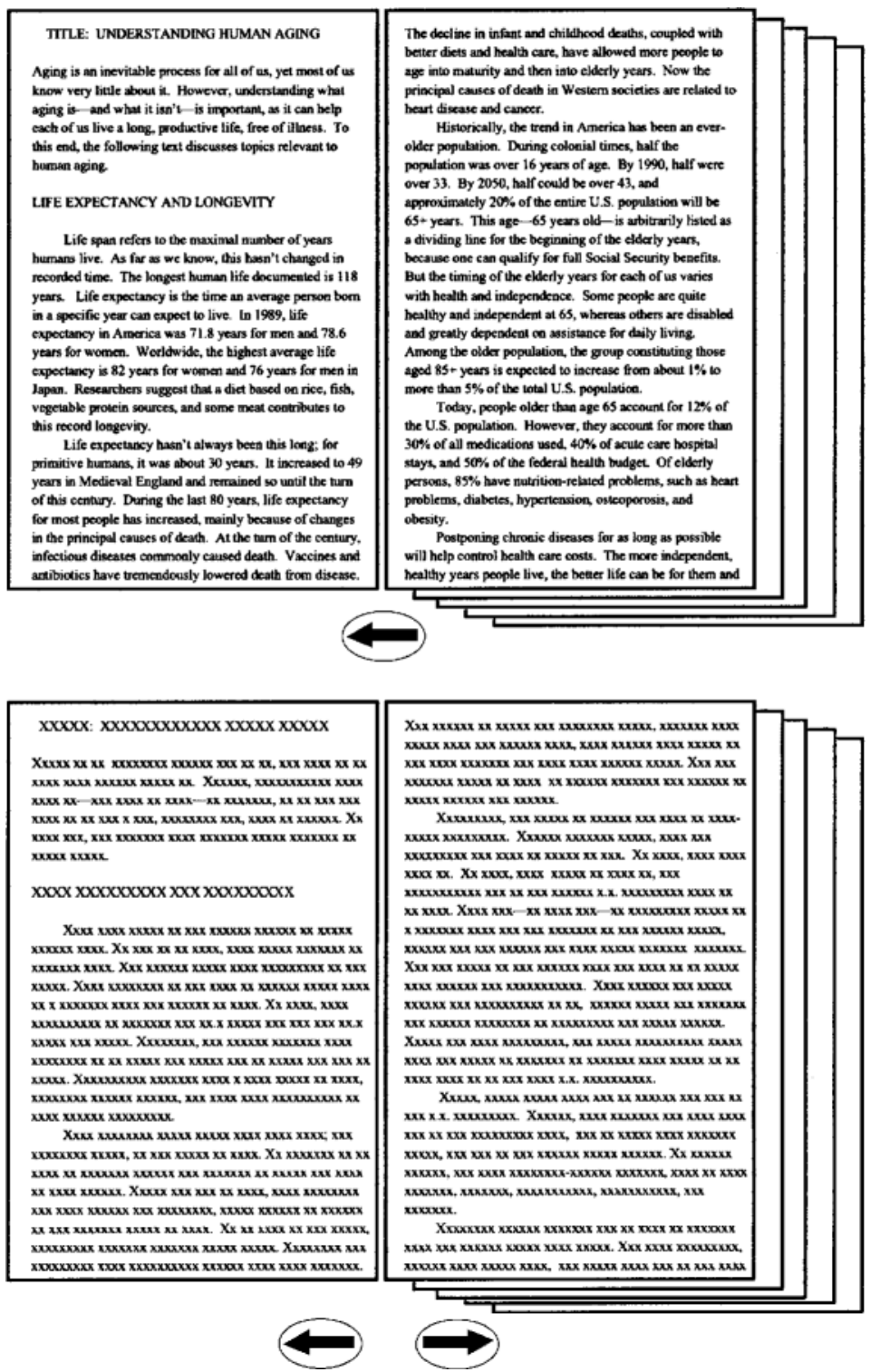

Figure 1. The top panel displays a miniature version of the virtual book used to present the experimental text for study, with the book "opened" to the first two pages. The bottom panel displays how the virtual book appeared with masked text during the relocation test.

asked to draw the original figure from memory. The paper folding task (Ekstrom et al., 1976) consisted of a total of 20 problems, each of which portrayed a series of line drawings depicting various folds made in a square piece of paper and then the punching of a hole through the folded paper. The participants were to select which of five drawings showed where the holes would be located when the paper was unfolded. The participants were given $6 \mathrm{~min}$ to complete the problems.

\section{Criterion Visuospatial Task}

We also administered the building location memory test (Ekstrom et al., 1976) as a criterion visuospatial task to check the predictive power of the visuospatial ability measures in case relocation performance did not correlate with them. This test was considered appropriate for this purpose because, just like the relocation task, it also required participants to recall specific spatial location information after some retention interval.

Each of two parts for the building location memory test began with a 4-min study trial of a simple street map that included line drawings of 12 different buildings. The participants were then given a test sheet that presented the same street map, except that each of the 12 buildings appeared in the margin rather than on the map. They were given 4 min to indicate in which sector of the map each building had appeared.

\section{Overall Procedure}

Each participant was tested in two sessions. The first session began with the relocation task, followed by Nelson-Denny Reading Test, 
building location memory test, study of the text for the narrative recall task, paper folding test, free recall of the narrative text, Corsi blocks task, and vocabulary test. In the second session, the participants performed the following tasks: copying of the Rey-Osterreith figure, the reading span task, and the redrawing of the figure from memory.

\section{Data Scoring}

Three scores were derived for the relocation task. The fill-in-theblank score was the total number of blanks filled in correctly (i.e., verbatim). Partial credit was given for items requiring multipleword responses if one of the words typed in was correct. The page score and line score were, respectively, the percentage of test items for which the correct page and the correct line in the original text were selected. A line selection was counted as correct if the selected line contained the spatial location of any part of the sentence in the test item, regardless of whether the correct page had been selected. Chance-level performance was $8 \%$ for the page score (based on 12 pages of text) and $10 \%$ for the line score (based on 30 lines per page and target sentences averaging 3 lines in length). We also calculated page and line scores on the basis of mean absolute distance (i.e., how many pages/lines they were away from the correct page/line), but because they showed qualitatively the same results, we report only the data based on the percentage correct measures below.

The reading span and Corsi block scores were the total number of target items recalled on trials in which perfect recall was obtained. The scores for the Nelson-Denny, vocabulary, paper folding, and building location memory tests were the total number of questions answered correctly within the time limits. The score for narrative recall was based on how many idea units (out of 40) were recalled verbatim or closely paraphrased. The score for the ReyOsterreith test was the number of elements (out of 18) in the original figure reproduced correctly.

To obtain a stable assessment of participants' verbal and visuospatial abilities, we derived aggregate ability scores. Specifically, scores on individual measures were first transformed into $z$ scores, and the mean of the transformed scores was then computed for each participant for each ability domain.

\section{RESULTS}

Table 1 presents the descriptive statistics for the main dependent measures. The mean fill-in-the-blank score was 54\%. More important, the mean page score and line score were $26 \%$ and $19 \%$, respectively. Consistent with the previous findings, they were both significantly greater than chance (chance level $=8 \%$ and $10 \%$, respectively) $[t(57)=10.74$ for page score, and $t(57)=7.13$ for line score; both $p$ s <.001].

Table 1 also presents the zero-order correlations among the main dependent measures. Both page and line scores were significantly correlated with the verbally based fill-in-the-blank score, whereas neither page nor line score was correlated with the criterion visuospatial task, building location memory. Interestingly, although both page and line scores were correlated moderately strongly with the verbal aggregate score $(r=.64$ and .41 , respectively), their correlations with the visuospatial aggregate score were rather modest ( $r=.23$ and .15$)$ and were significantly weaker than the corresponding correlations with the verbal aggregate score $[t(55)=3.21, p<$ .05 , for the page score, and $t(55)=1.75, p<.10$, for the line score].

Because the verbal and visuospatial aggregate scores showed a small yet significant correlation $(r=.28)$, we performed multiple regression analyses in which the verbal and visuospatial aggregate scores were simultaneously entered as predictor variables. Because this analysis controlled for the influence of the other variable, any observed relationships between the aggregate scores and dependent measures should reflect the contribution of cognitive factors that were more specifically verbal or visuospatial. The results of this analysis are summarized in Table 2.

As expected, only the verbal aggregate score was predictive of the fill-in-the-blank score, whereas only the visuospatial aggregate score was predictive of performance on the building location memory task, after we had controlled for the other variable. Of greater interest was the analysis involving the page and line scores. The verbal aggregate score was still highly predictive of both page and line scores (standardized coefficient $\beta=.62$ and .40 , respectively), even when visuospatial abilities were controlled for. In contrast, the visuospatial aggregate score was not predictive of either page or line score

Table 1

Descriptive Statistics and Zero-Order Correlations Between Dependent Measures and Aggregates of Individual Difference Measures $(N=\mathbf{5 8})$

\begin{tabular}{llrrrrr}
\hline & 1 & 2 & 3 & 4 & 5 & 6 \\
\hline 1. Fill-in-the-blank score in relocation task & \multicolumn{1}{c}{-} & & & & & \\
2. Page score in relocation task & $.46^{*}$ & - & & & \\
3. Line score in relocation task & $.41^{*}$ & $.68^{*}$ & - & & \\
4. Verbal aggregate score & $.61^{*}$ & $.64^{*}$ & $.41^{*}$ & - & \\
5. Visuospatial aggregate score & $.22^{\dagger}$ & $.23 \dagger$ & .15 & $.28^{*}$ & - & \\
6. Building location memory & .04 & .09 & -.16 & .17 & $.44^{*}$ & - \\
Mean & 54 & 26 & 19 & 0 & 0 & 19 \\
Standard deviation & 19 & 13 & 10 & .68 & .68 & 4 \\
Minimum & 14 & 4 & 4 & -1.33 & -1.40 & 3 \\
Maximum & 82 & 63 & 48 & 2.09 & 1.84 & 24 \\
\hline
\end{tabular}

${ }^{*} p<.05 . \dagger p<.10$. Descriptive statistics for fill-in-the-blank score, page score, and line score are reported as percentages. Descriptive statistics for aggregate scores are reported as aggregate $z$ scores, whereas those for building location memory are reported as number correct (out of 24). 
Table 2

Statistical Results of Simultaneous Regressions of Aggregate Measures on Dependent Variables

\begin{tabular}{|c|c|c|c|c|c|c|c|}
\hline \multirow[b]{3}{*}{ Dependent Variables } & \multicolumn{6}{|c|}{ Predictor Variables } & \multirow[b]{3}{*}{$R^{2}$} \\
\hline & \multicolumn{3}{|c|}{ Verbal Aggregate } & \multicolumn{3}{|c|}{ Visuospatial Aggregate } & \\
\hline & $\beta$ & $F$ & $p$ & $\beta$ & $F$ & $p$ & \\
\hline Fill-in-the-blank score & .59 & 28.31 & $<.001$ & .05 & $<1$ & n.s. & 0.37 \\
\hline Page score & .62 & 32.83 & $<.001$ & .06 & $<1$ & n.s. & 0.41 \\
\hline Line score & .40 & 9.98 & $<.01$ & .03 & $<1$ & n.s. & 0.17 \\
\hline Building location memory & .05 & $<1$ & n.s. & .43 & 11.44 & $<.001$ & 0.20 \\
\hline
\end{tabular}

Note-n.s. $=$ not significant at $p<.05 . \beta=$ standardized regression coefficient.

when verbal abilities were controlled for $(\beta=.06$ and .03 , respectively). Note that the latter finding cannot be attributed to the lack of predictive strength of the visuospatial aggregate score, given that it was a good predictor of building memory performance. Thus, these results strongly suggest that relocation of text information depends primarily on verbal, not visuospatial, abilities.

\section{DISCUSSION}

Intuitively, the relocating of text information would seem to depend primarily on visuospatial abilities. In the present study, however, the relationship between visuospatial abilities and relocation performance was tenuous at best, and it almost entirely disappeared after we had controlled for the effect of verbal abilities, which suggests that visuospatial abilities play a much lesser role in relocation than has commonly been assumed. Consistent with this view, Zechmeister et al. (1975) found that on only $4 \%$ of relocation decisions did readers report having a mental picture of the page and the specific page corner with the answer. Thus, although we sometimes have the experience of "seeing" the location of text content, the contribution of visuospatial abilities to information relocation appears to be minimal.

Why is the contribution of visuospatial abilities to relocation performance so small? One likely reason has to do with the transience of spatial location information. Although some spatial location information may be routinely encoded during reading (for a review, see Fischer, 2000), most such information seems to be lost rather quickly, perhaps within a few seconds in the case of word location memory (Fischer, 1999). Given that the time scale for the relocation task is at the level of minutes, not seconds, and that the goal of readers during study is to understand text content, rather than to remember its spatial layout, most of the encoded spatial location information may no longer be available by the time participants start the relocation phase of the task. Of course, location information for visually salient "landmarks" (such as headings, pictures, graphs, and special fonts) may be more enduring and could serve as useful clues for relocation performance, but such salient information is likely available to most individuals shortly after they have read a text, regardless of their visuospatial abilities. To the extent that this is indeed the case, the transient na- ture of spatial location information during reading may explain the minimal contribution of visuospatial abilities to relocating text information.

The present results instead point to verbal abilities as a major determinant of relocation performance. One plausible mechanism, outlined briefly earlier, is that verbal abilities support the encoding of text content into a detailed and coherent representation, which in turn may be used to infer the location of previously read text information. In other words, despite its seemingly visuospatial nature, relocating text information may primarily be a nonspatial inference process in which relevant spatial location information is "reconstructed" mainly if not exclusively on the basis of useful clues (e.g., the order in which various ideas have been presented in the text) derived from an individual's mental representation of the text. From this perspective, higher verbal abilities mean better skills at constructing a text representation and therefore better performance at relocating text information. Although this interpretation is admittedly speculative, it is consistent with Fischer's (1999) recent claim that readers use temporal and item information to reconstruct the spatial location of a word during sentence processing. Moreover, it can also easily accommodate the finding that when looking back in text to locate target information, poor readers reread indiscriminately whereas good readers are more selective (Zabrucky \& Commander, 1993).

This "verbally based reconstruction" view also provides a new account of the robust finding that relocation decisions are more accurate for correctly answered content questions than for incorrectly answered questions (Lovelace \& Southall, 1983; Rothkopf, 1971; Zechmeister \& McKillip, 1972; Zechmeister et al., 1975), a finding also replicated in the present study. The page and line scores were $29 \%$ and $21 \%$, respectively, for correctly answered fill-in-the-blank questions in this study, whereas they were $22 \%$ and $15 \%$, respectively, for incorrectly answered fill-in-the-blank questions. Although the differences were relatively small, both page and line scores were significantly greater for correctly answered than for incorrectly answered items $[t(57)=3.19$ and 3.19 , respectively, $p$ s $<.01]$.

In previous explanations of this effect (see, e.g., Lovelace \& Southall, 1983; Rothkopf, 1971), it has been assumed that relocation decisions are supported by 
visuospatial information, whereas answers to content questions are supported by verbal (or propositional) information, hence invoking two separate representations to explain the effect. An alternative explanation suggested by the present findings is that individuals rely at least in part on the same verbal representation to answer text questions and to infer the location of the information. In other words, text content that is better learned supports not only more correct responses to content questions but also more accurate inferences about text information location.

This alternative and more parsimonious account can even make new predictions. For example, according to this account, manipulations that improve textual coherence and thereby facilitate initial learning of the text should also improve the accuracy of location decisions (Christie \& Just, 1976; cf. Klusewitz \& Lorch, 2000). The "virtual book" methodology used in the present study should serve as a useful tool for evaluating this and other predictions and will likely provide further insights into the nature of representations and cognitive abilities underlying relocation performance.

\section{REFERENCES}

Baccino, T., \& Pynte, J. (1994). Spatial coding and discourse models during text reading. Language \& Cognitive Processes, 9, 143-155.

Baccino, T., \& Pynte, J. (1998). Spatial encoding and referential processing during reading. European Psychologist, 3, 51-61.

Brown, J. I., Bennett, J. M., \& Hanna, G. (1981). Nelson-Denny reading test. Chicago: Riverside.

Carroll, J. B. (1993). Human cognitive abilities: A survey of factoranalytic studies. New York: Cambridge University Press.

Christie, J. M., \& Just, M. A. (1976). Remembering the location and content of sentences in a prose passage. Journal of Educational Psychology, 68, 702-710.

Daneman, M., \& Carpenter, P. A. (1980). Individual differences in working memory and reading. Journal of Verbal Learning \& Verbal Behavior, 19, 450-466.

Ekstrom, R. B., French, J. W., Harman, H. H., \& Derman, D. (1976). Kit of factor-referenced cognitive tests. Princeton, NJ: Educational Testing Service.

Fischer, M. H. (1999). Memory for word locations in reading. Memory, 7, 79-116.

FISCHER, M. H. (2000). Perceiving spatial attributes of print. In A. Kennedy, R. Radach, D. Heller, \& J. Pynte (Eds.), Reading as a perceptual process (pp. 89-117). New York: Elsevier.

FrAZIER, L., \& RAYNER, K. (1982). Making and correcting errors during sentence comprehension: Eye movements in the analysis of structurally ambiguous sentences. Cognitive Psychology, 14, 178-210.

Kennedy, A., \& Murray, W. S. (1987). Spatial coordinates and reading: Comments on Monk (1985). Quarterly Journal of Experimental Psychology, 39A, 649-656.

KLUSEWITZ, M. A., \& LORCH, R. F., JR. (2000). Effects of headings and familiarity with a text on strategies for searching a text. Memory \& Cognition, 28, 667-676.

Lovelace, E. A., \& Southall, S. D. (1983). Memory for words in prose and their locations on the page. Memory \& Cognition, 11, 429434.

Miyake, A., Friedman, N. P., Rettinger, D. A., Shah, P., \& HegARTY, M. (2001). How are visuospatial working memory, executive functioning, and spatial abilities related? A latent variable analysis. Journal of Experimental Psychology: General, 130, 621-640.

Rотнкорғ, E. Z (1971). Incidental memory for location of information in text. Journal of Verbal Learning \& Verbal Behavior, 10, 608-613.

Shah, P., \& MiYAKe, A. (1996). The separability of working memory resources for spatial thinking and language processing: An individual differences approach. Journal of Experimental Psychology: General, 125, 4-27.

Spreen, O., \& Strauss, E. (1998). A compendium of neuropsychological tests (2nd ed.). New York: Oxford University Press.

ZABRUCKy, K., \& Commander, N. E. (1993). Rereading to understand: The role of text coherence and reader proficiency. Contemporary Educational Psychology, 18, 442-454.

Zechmeister, E. B., \& McKillip, J. (1972). Recall of place on the page. Journal of Educational Psychology, 63, 446-453.

Zechmeister, E. B., McKillip, J., Pasko, S., \& Bespalec, D. (1975). Visual memory for place on the page. Journal of General Psychology, 92, 43-52.

\section{APPENDIX \\ Sample Excerpt from Experimental Text \\ and Sample Test Items}

\section{Text Excerpt}

... When researchers closely examine the genetic and lifestyle backgrounds of long-lived people, they surmise that vigorous physical activity, low-fat diets, and the prevention of excessive weight gain may be key factors of longevity. Studies of families, and of twins in particular, also provide evidence for genetic control of human longevity. Identical twins tend to have very similar life spans and causes of death. Because identical twins have exactly the same genetic information, this argues strongly that longevity is determined at least partially by heredity.

Animal studies tell us something about longevity. By limiting energy intake to about $60 \%$ of usual in rodents, life span can be increased by $35 \%$, with at least $50 \%$ lower incidence of cancer. Only energy should be limited; the rodent diet is supplemented with vitamins and minerals. Some studies have shown that food restriction can be started later in life and still extend the life span of rats ...

\section{Sample Short-Answer Test Items}

1._____________________ to have very similar life spans and causes of death. (identical twins)

2. By limiting energy intake to about $60 \%$ of usual in rodents, life span can be increased by $35 \%$, with at least $50 \%$ lower incidence of . (cancer) 